\title{
Josephson Effect for Superconductors Lacking Time-Reversal and Inversion Symmetries
}

\author{
B. Leridon, ${ }^{1}$ Tai-Kai Ng, ${ }^{2}$ and C. M. Varma ${ }^{3}$ \\ ${ }^{1}$ UPR5, CNRS/ESPCI, 10 rue Vauquelin, 75231 Paris cedex 05, France \\ ${ }^{2}$ Department of Physics, HKUST, Hong Kong \\ ${ }^{3}$ Department of Physics, University of California, Riverside, California 92521, USA
}

(Received 4 April 2006; published 12 July 2007)

\begin{abstract}
Because of the absence of a center of inversion in some superconducting compounds, a $p$-wave admixture to the dominant $d$-wave (or $s$ ) order parameter must exist. If time reversal is also violated, an allowed invariant is the product of the $d$ wave (or $s$ wave), $p$ wave, and an appropriately directed current. We show that this leads to a new and remarkable property of the Josephson current for tunneling into a $s$-wave superconductor along the direction parallel to the axis of the $p$-wave component. These ideas are applied to the heavy-fermion compounds which lack center of inversion due to crystalline symmetry, as well as time-reversal symmetry, such as $\mathrm{CePt}_{3} \mathrm{Si}$. They also apply to the superconducting state of the cuprates in the pseudogap region of the phase diagram where in the normal phase some experiments have detected a time-reversal and inversion symmetry broken phase.
\end{abstract}

DOI: 10.1103/PhysRevLett.99.027002

A microscopic state violating time-reversal and inversion symmetry has been proposed for the pseudogap state of the cuprates [1]. The microscopic ground state associated with such symmetry breaking has spontaneous current loops in the $\mathrm{O}-\mathrm{Cu}-\mathrm{O}$ plaquettes in each cell from zero temperature all the way to the pseudogap temperature $T^{*}$. Two classes of experiments (ARPES with circularly polarized photons [2,3] and polarized neutron diffraction [4]) on two different cuprate compounds have observed such a state below $T^{*}$. These broken symmetries are expected to continue into the superconducting state, as indeed found in the experiments $[2,4]$. This should then also affect the symmetry of superconductivity $[5,6]$.

Some heavy-fermion superconductors such as $\mathrm{CePt}_{3} \mathrm{Si}$ lack center of inversion in their crystal structure and similar superconducting states are expected for them [7,8]. $\mathrm{CePt}_{3} \mathrm{Si}$ is noncentrosymmetric, antiferromagnetic below $2.2 \mathrm{~K}$, and superconducting below $0.75 \mathrm{~K}$. There is an ongoing debate about its order parameter symmetry [7,9], and the possibility of mixed spin-triplet and spin-singlet pairing has been suggested. Thermal conductivity measurements have demonstrated the existence of line nodes [10]. The relatively high value of $H_{c 2}(5 \mathrm{~T})$ (above the Pauli-Clogston limit) seems to indicate a spin-triplet superconductor.

Here we will show that the Josephson effect between a superconductor breaking inversion symmetry and timereversal symmetry and a conventional $s$-wave superconductor has a distinct signature from conventional Josephson effect. The clear observation of such effects will further substantiate the nature of the pseudogap phase for the cuprates and the nature of superconductivity in the relevant heavy-fermion compounds.

Quite generally, if time-reversal and inversion symmetry are broken, a term in the free-energy of the form
PACS numbers: 74.50.+r, 74.20.Rp, 74.72.-h, 74.70.Tx

$$
i \epsilon\left(\psi_{d}^{*} \psi_{p x}-\text { c.c. }\right)
$$

is allowed. $\psi_{d}$ is the dominant " $d$-wave" superconducting order parameter, $\psi_{p x}$ is a " $p$-wave" order parameter, and the coefficient $\epsilon$ is proportional to the order parameter associated to the symmetry breaking in the normal phase (for example, the crystal structure lacking center of inversion [7] or the spontaneous current loops [1] in cuprates). $x$ corresponds to the (110) direction or the (110) direction depending on the domain for the cuprates, and to the $c$ axis for $\mathrm{CePt}_{3} \mathrm{Si}$. (We have introduced $i$ explicitly in the coefficient so that $\epsilon$ is real.) In this case a superconducting state violating time-reversal and inversion symmetry and consistent with the proposed normal state is of the generic form $\psi\left(d_{x y}\right)+i \delta_{0} \psi\left(p_{x, y}\right)$ in zero magnetic field. $\delta_{0}=$ $\epsilon / 2 \alpha_{d}$, where the leading term in the free energy for the $d$-wave component is $\alpha_{d}\left|\psi_{d}\right|^{2}$. Such states have actually been investigated in the context of heavy fermions materials whose crystal structure lacks inversion symmetry $[7,8]$ and some experiments have already been proposed to discover evidence for such a superconducting state in the cuprates [5].

Consider a surface of the superconductor perpendicular to the $x$ direction. Then for the state $\psi\left(d_{x y}\right)+i \delta_{0} \psi\left(p_{x}\right)$, spontaneous supercurrent flows in the $y$ direction, i.e., along the surface. The alternative state $\psi\left(d_{x y}\right)+$ $i \delta_{0} \psi\left(p_{y}\right)$ has current normal to the surface and is not allowed. So the presence of a surface whose normal is the $x$ direction will naturally favor the $p_{x}$ admixture. In the cuprates, such a surface also generates Andreev bound states (ABS) whose consequences have been widely investigated [11,12]. The effect we are discussing here is quite distinct and leads to rather different predictions. The consequence on the zero bias anomaly due to the ABS of such a superconducting state has been discussed elsewhere [5]. 
Let us now consider that on this surface, a small junction $\left(L \ll \lambda_{J}\right)$ is fabricated which allows a Josephson coupling to a conventional $s$-wave superconductor. We now wish to consider the modification of the superconducting state due to an applied magnetic field in a direction in the plane of the junction. This modification must come about because beside the invariant of Eq. (1), there also exists an invariant proportional to $i j_{y} \psi\left(p_{x}\right) \psi\left(d_{x y}\right)$. This is because $j_{y}$, the current in the $y$ direction, is odd under time inversion [as is $\left.i \psi\left(p_{x}\right)\right]$ and the product of the three terms satisfies all inversion and reflection invariances. Such a term can be derived from the Ginzburg-Landau free energy for the problem as follows.

Let the magnetic field $\mathbf{B}$ be in the $\hat{\mathbf{z}}$ direction and adopt the London gauge in which the vector potential is

$$
\mathbf{A}=B x \hat{\mathbf{y}}
$$

The free-energy density may then be written as

$$
\begin{gathered}
F\left(\psi\left(d_{x y}\right), \psi\left(p_{x}\right), A\right)=\alpha_{d}\left|\psi\left(d_{x y}\right)\right|^{2}+K\left|\vec{D} \psi\left(d_{x y}\right)\right|^{2} \\
+\beta_{d}\left|\psi\left(d_{x y}\right)\right|^{4}+\alpha_{p}\left|\psi\left(p_{x}\right)\right|^{2} \\
+i \epsilon\left[\psi\left(d_{x y}\right)^{*} \psi\left(p_{x}\right)-\psi\left(d_{x y}\right) \psi\left(p_{x}\right)^{*}\right] \\
+i \epsilon^{\prime}\left[\psi\left(d_{x y}\right)^{*} D_{y} \psi\left(p_{x}\right)-\psi\left(d_{x y}\right) D_{y}^{*} \psi\left(p_{x}\right)^{*}\right],
\end{gathered}
$$

where $\vec{D}=-i(\nabla-2 e i \vec{A} / \hbar c)$. This is simply the modification of the free energy written down by Agterberg and Kaur [6] for the present geometry and in the presence of a magnetic field. $\epsilon$ has already been discussed; $\epsilon^{\prime}$ has a magnitude of the order of the Fermi velocity and is independent of the underlying inversion or time-reversal breaking order parameter. On minimizing with respect to both the magnitudes and phases of $d_{x y}$ and $p_{x}$, one obtains that the order parameter is

$$
\psi=\psi\left(d_{x y}\right)+i \delta \psi\left(p_{x}\right),
$$

where

$$
\begin{aligned}
\psi\left(d_{x y}\right) & =\left|\psi\left(d_{x y}\right)\right| \exp \left(i \theta_{0 x}+i \phi_{B}(x)\right), \\
\phi_{B}(x) & =\frac{2 e}{\hbar} y \int^{x} B\left(x^{\prime}\right) d x^{\prime}
\end{aligned}
$$

and

$$
\delta=\left[\delta_{0}-\zeta j_{y}\right] \Delta_{d} \operatorname{expi} \phi_{B}(x) .
$$

Here $\Delta_{d}$ is the magnitude of the major order parameter, $\theta_{0 x}=x \epsilon \epsilon^{\prime} / K^{R}$ specifies the spiral state deduced by Agterberg and Kaur [6], $\delta_{0}=\left[\epsilon\left(1+\epsilon^{\prime 2} / K^{R}\right)\right]$ is the magnitude of the bulk induced $p$-wave order parameter, and $K^{R}=K-\epsilon^{\prime 2} / \alpha_{p}$ and $\zeta=\epsilon^{\prime} /\left(2 \alpha_{p} \Delta_{d}^{2} K^{R}\right)$. The length associated with the pitch of the spiral can be estimated to be much larger than the London penetration depth $\lambda$. The spiral then produces negligible effects in Josephson tunneling and will be ignored by putting $\theta_{0}=0$.

The second part of Eq. (7) is equivalent to having the aforementioned term in the free-energy proportional to $i j_{y} \psi\left(p_{x}\right) \psi\left(d_{x y}\right)$. There are two contributions to $j_{y}$. One is just the Meissner screening current, which is uniform on the surface,

$$
j_{y}^{M}=\frac{-c B}{4 \pi \lambda} \exp (-x / \lambda) .
$$

The other arises when a Josephson junction is constructed of this superconductor with another superconductor (of, for example, the $s$-wave kind) and a magnetic field is applied in the junction as above. Then, the Josephson current density across the tunnel junction is periodic in $y$,

$$
j_{x}^{J}(y)=J_{x 0} \sin \left(2 \pi B w y / \Phi_{0}+\gamma_{0}\right) .
$$

Here $w$ is the effective thickness of the barrier in the $x$ direction and $\gamma_{0}$ is the phase difference across the barrier. It then follows, by continuity or by considering the gradient of the phase on the surface of either superconductor, that there exists also a periodic current along the $y$ direction as in every Josephson junction. Usually, in a small Josephson junction (the case considered here) it has no observable consequence. In our case of the $d+i p$ superconductor, it changes the magnitude of the $p_{x}$-wave component periodically in the $y$ direction. Note that since this modulation is itself proportional to the Josephson current, it will have an effect on the Josephson current which is second order in the tunneling. We mention it for completeness although it has a negligible effect on our results and is dropped below.

Collecting Eq. (5)-(8), the wave function of the $d+i p$ superconductor near the surface of the Josephson junction up to a depth about $\lambda$ in $x$ direction is

$$
\begin{aligned}
\psi(y) & =\Delta_{d}\left[f_{d}(\theta)+i \delta^{*} \cos (\theta)\right] \exp \left[i \phi_{B}(y)\right] \\
& =\Delta \exp \left\{i\left[\phi_{B}(y)+\phi(y)\right]\right\},
\end{aligned}
$$

where

$$
\begin{gathered}
\Delta=\Delta_{d} \sqrt{f_{d}^{2}(\theta)+\delta^{* 2} \cos ^{2}(\theta)} \\
\phi=\arctan \left[\delta^{*} \cos \theta / f_{d}(\theta)\right] \\
\delta^{*}=\left(\delta_{0}+\delta_{1} B\right), \quad \delta_{1}=\zeta c /(4 \pi \lambda), \quad f_{d}(\theta)=\sin (2 \theta) .
\end{gathered}
$$

We may now calculate the Josephson current between the $d+i p$ superconductor oriented along the $x$ direction and a conventional $s$-wave superconductor.

$$
I_{J} \propto \int_{-\pi / 2}^{\pi / 2} d \theta F(\theta) \int_{-L / 2}^{L / 2} d y \Delta \sin \left[\gamma_{0}+\frac{2 e}{\hbar} B \lambda y+\phi\right] .
$$

$F(\theta)$ gives the tunneling cone and $L$ is the length of the 
barrier in the $y$ direction. We can evaluate the integral to get

$$
\begin{aligned}
I_{J}= & A_{1} \int_{-L / 2}^{L / 2} d y\left\{\sin \left[\gamma_{0}+\left(2 \pi B \lambda_{L} / \Phi_{0}\right) y\right]\right\} \\
& +A_{2} \int_{-L / 2}^{L / 2} d y\left\{\delta \cos \left[\gamma_{0}+\left(2 \pi B \lambda_{L} / \Phi_{0}\right) y\right]\right\},
\end{aligned}
$$

where $\quad A_{1}=\Delta_{0} \int_{-\pi / 2}^{\pi / 2} d \theta F(\theta) f_{d}(\theta) \quad$ and $\quad A_{2}=$ $\Delta_{0} \int_{-\pi / 2}^{\pi / 2} d \theta F(\theta) \cos (\theta)$. We obtain

$$
\begin{aligned}
\frac{I_{J}}{L}= & A_{1} \sin \left(\gamma_{0}\right) \frac{\Phi_{0}}{\pi \Phi} \sin \left(\frac{\pi \Phi}{\Phi_{0}}\right) \\
& +A_{2}\left[\left(\delta_{0}+\delta_{1} B\right) \cos \left(\gamma_{0}\right) \frac{\Phi_{0}}{\pi \Phi} \sin \left(\frac{\pi \Phi}{\Phi_{0}}\right)\right]
\end{aligned}
$$

$\Phi=B \lambda_{L} L$ is the total flux through the junction. This expression should be maximized with respect to $\gamma_{0}$ to get the observable Josephson current. The $I_{J}$ thus obtained is

$$
\frac{I_{J}}{L}=\left[A_{1}^{2}+A_{2}^{2}\left(\delta_{0}+\delta_{1} B\right)^{2}\right]^{1 / 2}\left|\frac{\Phi_{0}}{\pi \Phi} \sin \left(\frac{\pi \Phi}{\Phi_{0}}\right)\right| .
$$

The first term in $I_{J}^{2}$ is the usual term. It would be zero in a tetragonal crystal where $f_{d}(\theta)=\sin (2 \theta)$, assuming a very narrow tunnel cone. The second term has two contributions. The more interesting part, arising from the Meissner current, is simply proportional to $\sin \left(\frac{\pi \Phi}{\Phi_{0}}\right)$ because the factor $B$ in the numerator cancels the factor $B$ in the flux $\Phi$ in the denominator. This last feature is remarkable since it leads to a nondecreasing amplitude for the Josephson currents with magnetic field for large fields [13].

The results of the evaluation of Eq. (17) are represented in Fig. 1 for a conventional $d$ or $s$ or $d+s$ material (red dotted line), and a $d+i p$ material (blue solid line). The main observation is that the critical current does not decrease at large fields but oscillates. For this calculation it has been assumed that a single domain of the $p$-wave component exists over the entire length $L$ of the junction.

However, in real crystals, $d+i p$ domains are expected to occur, whenever the $p_{x}$ component changes sign with respect to the $d$ component, which introduces two other terms that were previously canceled when integrating over the junction. Let us consider $n_{\max }$ randomly distributed domains along the $y$ direction. Each domain extends from $\frac{l_{n}}{L}$ to $\frac{l_{n+1}}{L}$. Each domain boundary corresponds to a change of sign of the $p_{x}$ component. In the expression for the current Eq. (16), the term proportional to $A_{2}$ is replaced by

$$
-A_{2} \cos \left(\gamma_{0}\right) \sum_{n=0}^{n_{\max }-1}(-1)^{n+1} \times\left(\delta_{0}+\delta_{1} B\right)\left\{\frac{\Phi_{0}}{2 \pi \Phi}\left[\cos \left(\frac{2 \pi \Phi \ell_{n+1}}{\Phi_{0} L}\right)-\cos \left(\frac{2 \pi \Phi \ell_{n}}{\Phi_{0} L}\right)\right]+\frac{\Phi_{0}}{\pi \Phi}\left[\sin \left(\frac{\pi \Phi \ell_{n+1}}{\Phi_{0} L}\right)-\sin \left(\frac{\pi \Phi \ell_{n}}{\Phi_{0} L}\right)\right]\right\} .
$$

The results of the calculation for multidomains samples are presented on Fig. 2 for two domains in two different configurations (red dotted line and blue dashed line) and 5

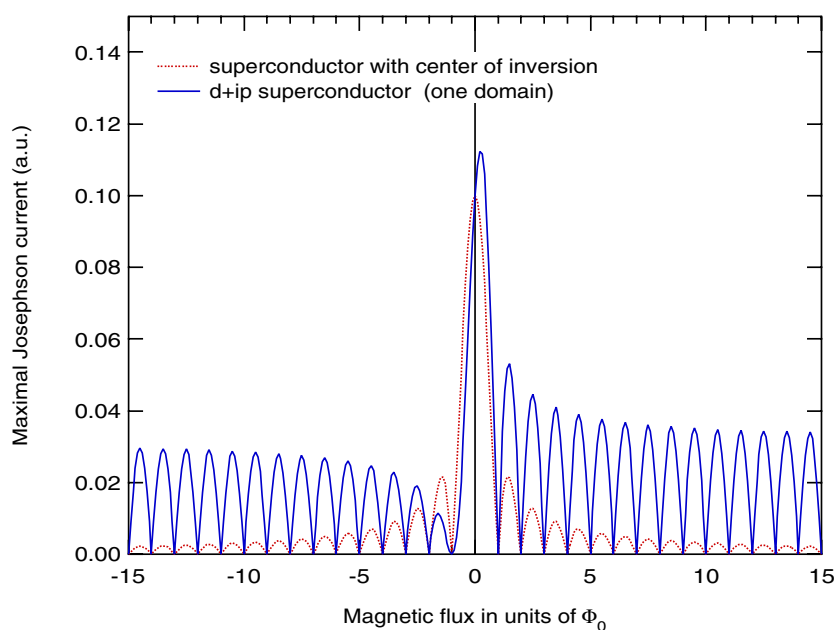

FIG. 1 (color online). Josephson current patterns as a function of magnetic field. Red dotted line: conventional Josephson pattern expected for a superconductor. Blue solid line: Josephson pattern expected for a single domain $d+i p$ superconductor. The parameters used for the calculation in reference to Eq. (17) are $A_{1}=0.02 \mathrm{Am}^{-1}, A_{2} \delta_{0}=0.1 \mathrm{~A} \mathrm{~m}^{-1}, A_{2} \delta_{1}=$ $0.05 \mathrm{~A} \mathrm{~m}^{-1} \mathrm{~T}^{-1}$. domains (black solid line). The usual Fraunhofer pattern is replaced by a complicated interference pattern. The pat-

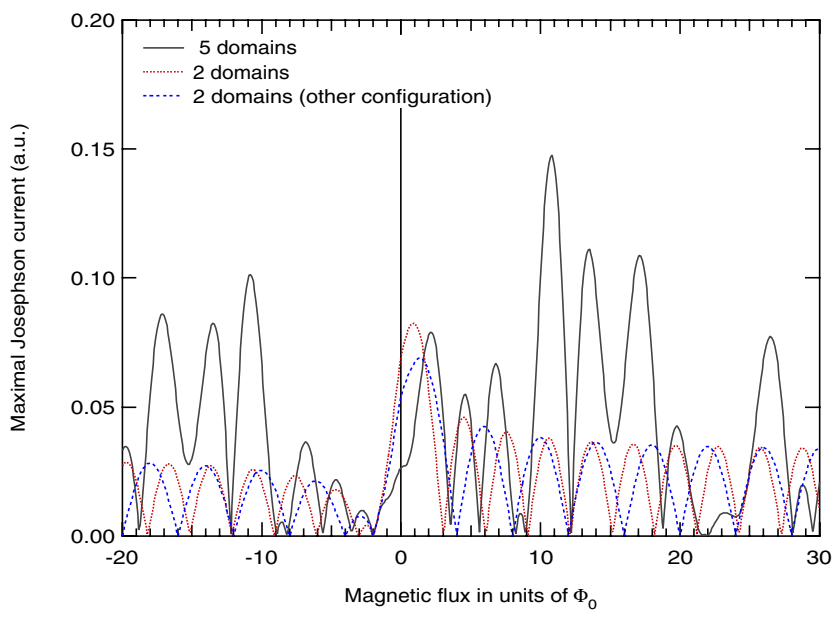

FIG. 2 (color online). Josephson current patterns as a function of magnetic field for a " $d+i p$ " superconductor with domains of the $p$-wave component. The parameters are the same as in Fig. 1. Red dotted and blue dashed curves are for two domains of different configuration whose borders are given by $(-L / 2$, $-L / 3,+L / 2)$ and $(-L / 2,+L / 4,+L / 2)$, respectively. The black solid curve is for five random domains $(-L / 2,-0.41 \mathrm{~L}$, $0.09 \mathrm{~L}, 0.15 \mathrm{~L}, 0.23 \mathrm{~L},+L / 2)$. Parameters are $A_{1}=0.02 \mathrm{~A} \mathrm{~m}^{-1}$, $A_{2} \delta_{0}=0.1 \mathrm{~A} \mathrm{~m}^{-1}, A_{2} \delta_{1}=0.05 \mathrm{~A} \mathrm{~m}^{-1} \mathrm{~T}^{-1}$. 
terns are not even in field and they do not show a decrease with magnetic flux; however, the periodicity of a flux quantum is still apparent. (The modification of the pattern due to neglected Josephson loop currents introduces a second harmonic but their magnitude is expected to be very small.) The observed experimental pattern should vary depending on the geometry of the domains, but in every case it should retain a component whose magnitude does not decrease with field at large fields.

When tunneling along the (001) direction of the cuprates, i.e., tunneling perpendicular to the $\mathrm{Cu}-\mathrm{O}$ planes, the $p$-order parameter with or without a magnetic field contributes no Josephson current. Therefore, in this geometry only a conventional Fraunhoffer pattern should be observed.

The situation is more complicated when tunneling along the 100 direction, i.e., along the lobes of the $d$-wave order parameter. A $p$-wave component would then be necessarily accompanied by a current at an angle $\pi / 4$ to the boundary. This is not allowed. A system of domains along the surface can be envisaged which cancel the component of the current perpendicular to the surface. We have not investigated the energetics of this possibility, but if this is realized, the Josephson current variation with a magnetic field is expected also not to be decaying as in a Fraunhoffer pattern, due to the boost of the $p$-wave by the Meissner current.

We are aware of one set of Josephson experiments in $\mathrm{YBa}_{2} \mathrm{Cu}_{3} \mathrm{O}_{6+x}$ with tunneling direction perpendicular to the layers (001) as well as along the (110) and (100) direction [14]. In the former the usual Fraunhofer diffraction pattern is indeed observed. In the latter, an oscillatory form remains at large $\Phi / \Phi_{0}$, which is consistent with the prediction here and qualitatively resembles the simulation on Fig. 2. The exact pattern is expected to depend on the precise geometry of the $d+i p$ domains and therefore is expected to vary only upon warming up and cooling down the sample which was indeed observed. However, in these experiments, it is not known (although probable) whether the thin films were underdoped. At a (110) surface in a $d$-wave superconductor, it is known that depairing occurs, zero-energy ABS $[11,15]$ are created, and the enhancement of a subdominant order parameter such as $d$ or $s$ (but not $p$ ) has been proposed [16]. The properties of the current associated to tunneling into those ABS have been theoretically investigated by [17]. However, only a $d+i p$ order parameter can produce the nondecreasing Josephson patterns described here. We suggest experiments in which the quasiparticle tunneling above $T_{c}$ should be monitored simultaneously with the Josephson tunneling below $T_{c}$ to see if the effects predicted here only occur in samples which show the pseudogap in the quasiparticle tunneling. This experiment, if verified, may constitute an additional observation of the symmetry breaking in the pseudogap phase of the cuprates.

The predictions here also have applications in appropriate geometries to other superconductors lacking a center of inversion [7]. Actually Josephson effect measurements of the kind we have proposed have already been done on the heavy-fermion compound $\mathrm{CePt}_{3} \mathrm{Si}$ [18]. Sumiyama et al. [18] have measured a conventional Josephson pattern in the $a$-axis direction, while along the $c$ axis, along which the mirror is lost, the patterns exhibit oscillating nondecreasing Josephson currents similar to our predictions. This may therefore constitute a specific proof that both inversion symmetry and time-reversal symmetry are lost in this compound and that the order parameter is indeed $d+i p$ in this material.

T.K.N. acknowledges support by HKRGC through Grant No. 602803.

[1] C. M. Varma, Phys. Rev. Lett. 83, 3538 (1999); Phys. Rev. B 73, 155113 (2006).

[2] A. Kaminski et al., Nature (London) 416, 610 (2002).

[3] M. E. Simon and C. M. Varma, Phys. Rev. Lett. 89, 247003 (2002).

[4] B. Fauque et al., Phys. Rev. Lett. 96, 197001 (2006).

[5] T. K. Ng and C.M. Varma, Phys. Rev. B 70, 054514 (2004).

[6] R.P. Kaur and D.F. Agterberg, Phys. Rev. B 68, 100506(R) (2003).

[7] E. Bauer et al., Phys. Rev. Lett. 92, 027003 (2004).

[8] R. P. Kaur, D. F. Agterberg, and M. Sigrist, Phys. Rev. Lett. 94, 137002 (2005).

[9] N. Metoki et al., J. Phys. Condens. Matter 16, L207 (2004).

[10] K. Izawa, Y. Kasahara. Y. Matsuda, K. Behnia, T. Yasuda, R. Settai, and Y. Onuki, Phys. Rev. Lett. 94, 197002 (2005).

[11] J. Lesueur, L. H. Greene, W. L. Feldmann, and A. Inam, Physica (Amsterdam) 191C, 325 (1992).

[12] T. Lofwander, V. S. Shumeiko, and G. Wendin, Supercond. Sci. Technol. 14, R53 (2001).

[13] The same behavior happens due to quite different physics and at a quite different period in squids.

[14] X. Grison, Ph.D. thesis, Ecole Polytechnique, 2000, http:// www. imprimerie.polytechnique.fr/Theses/Files/Grison.pdf.

[15] Chia-Ren Hu, Phys. Rev. Lett. 72, 1526 (1994).

[16] See Fogelström Phys. Rev. Lett. 79, 281 (1997), and references therein.

[17] Y. Tanaka and S. Kashiwaya, Phys. Rev. Lett. 74, 3451 (1995); Phys. Rev. B 56, 892 (1997); S. Kashiwaya and Y. Tanaka, Rep. Prog. Phys. 63, 1641 (2000).

[18] A. Sumiyama et al., J. Phys. Soc. Jpn. 74, 3041 (2005). 